\title{
Near-field multiple scattering effects of plasmonic nanospheres embedded into thin-film organic solar cells
}

\author{
Wei E. I. Sha, Wallace C. H. Choy, ${ }^{\text {a) }}$ Yang G. Liu, and Weng Cho Chew ${ }^{\text {b) }}$ \\ Department of Electrical and Electronic Engineering, The University of Hong Kong, Pokfulam Road, \\ Hong Kong
}

(Received 22 June 2011; accepted 20 August 2011; published online 14 September 2011)

\begin{abstract}
We investigate near-field multiple scattering effects of plasmonic nanospheres (NSPs) embedded into organic solar cells (OSCs). When NSPs are embedded into a spacer layer, the near-field scattering from the NSPs shows strong direction-dependent features, which significantly affects the optical absorption. When NSPs are embedded into an active layer, the absorption enhancement is attributed to the interplay between longitudinal and transverse modes supported by the NSP chain. The breakdown of electrostatic scaling law is confirmed by our theoretical model and should be accounted for optical designs of OSCs. The work provides the fundamental physical understanding and design guidelines for plasmonic photovoltaics. (C) 2011 American Institute of Physics. [doi:10.1063/1.3638466]
\end{abstract}

Thin-film solar cells $(\mathrm{SCs})^{1}$ play a central role in reducing the cost of traditional crystallized bulk SCs to compete with the fossil fuel. However, insignificant optical absorption resulting from the thin-film design is a tremendous problem for the SCs. Plasmons, ${ }^{2}$ due to their unique features of tunable resonance and near-field concentration for enhancing light trapping and harvesting the light absorption, become one of the best candidates for achieving both enhanced optical absorption and significant photocurrent carrier collection. Recently, the improved optical absorption or short-circuit current has been experimentally and theoretically demonstrated in thin-film SCs by using metallic nanospheres (NSPs), ${ }^{3-11}$ which can be explained by the plasmon-induced light-trapping and scattering effects. ${ }^{12}$ However, a deep physical understanding is still required to reexamine some fundamental concepts and unveil underlying device physics qualitatively and quantitatively.

In this work, we study in detail on the near-field multiple scattering effects of plasmonic NSPs embedded into thinfilm organic SCs (OSCs). A rigorous electrodynamic approach is developed to characterize the optical absorption of the OSC. The fundamental physics of the optical absorption shows remarkable differences between the NSPs embedded into a spacer and those embedded into an active layer. The direction-dependent features of near-field scattering from NSPs significantly affect the absorption enhancement when NSPs are embedded into the spacer. The interaction between longitudinal and transverse modes supported in the NSP chain plays a key role in the absorption enhancement when NSPs are embedded into the active layer. Through properly engineering the position and spacing of NSPs, our theoretical results show that the absorption enhancement can be improved by about $100 \%$. Moreover, we demonstrate the breakdown of the electrostatic scaling law that should be considered in the design of OSCs. The work provides the physical guidelines for plasmonic OSCs.

\footnotetext{
${ }^{\text {a)} E l e c t r o n i c ~ m a i l: ~ c h c h o y @ e e e . h k u . h k . ~}$

b) Also at Department of Electrical and Computer Engineering, University of Illinois, Urbana-Champaign, Illinois 61801, USA. Electronic mail: wcchew@hku.hk.
}

Figs. 1(a) and 1(b) illustrate a schematic pattern of a bulk heterojunction OSC nanostructure to be investigated. The active layer is a typical blend polymer of P3HT (poly(3hexylthiophene)) and PCBM (methanofullerene). A hole conduction layer is PEDOT:PSS (poly(3,4-ethylenedioxythiophene):poly(4-styrenesulfonic acid)) chosen as a spacer between an electrode and the active layer. Fig. 1(c) shows the absorption coefficient of the active material measured from ellipsometry. ${ }^{11}$ With tunable size and spacing, a spherical chain comprising multiple silver (Ag) NSPs is embedded into the spacer or active layer as near-field concentrators. The complex refractive index of $\mathrm{Ag}$ can be expressed with the Brendel-Bormann model. ${ }^{13}$ An incident light is propagated from the spacer to the active layer with a TM polarization $\left(H_{z}^{i}=0\right)$ at a vertical $\left(0^{\circ}\right)$ and an oblique $\left(60^{\circ}\right)$ incidences. In comparison with the TE polarization, the TM polarization supports much stronger dipole-dipole couplings between NSPs. Moreover, the absorption enhancement by a silver NSP array, which has a polarization-independent feature, can be regarded as a superposition of those by silver NSP chains with both the TM and TE polarizations. To unveil device-related multiple scattering mechanism of NSPs, we develop a rigorous electrodynamic approach to characterize the optical properties of the OSC. (see supplementary material.) ${ }^{14}$ The absorption spectrum of the OSC is calculated by

$$
A(\lambda)=\int n(\lambda) k(\lambda) \frac{2 \pi c}{\lambda} \epsilon_{0}|\mathbf{E}|^{2} d V
$$

where $n_{c}=n+i k$ is the complex refractive index of the active material, $\lambda$ is the incident wavelength, and $\epsilon_{0}$ is the permittivity of free space. It is worth mentioning that we only calculate the optical absorption of the active material excluding the metallic absorption of NSPs especially when NSPs are embedded into the active layer. Figs. 2 and 3 show the spectral enhancement factors, which are the absorption spectrums of the OSC incorporating NSPs over that excluding NSPs. Using standard solar irradiance spectrum (air mass 1.5 global), the total absorption of the OSC can be given by 

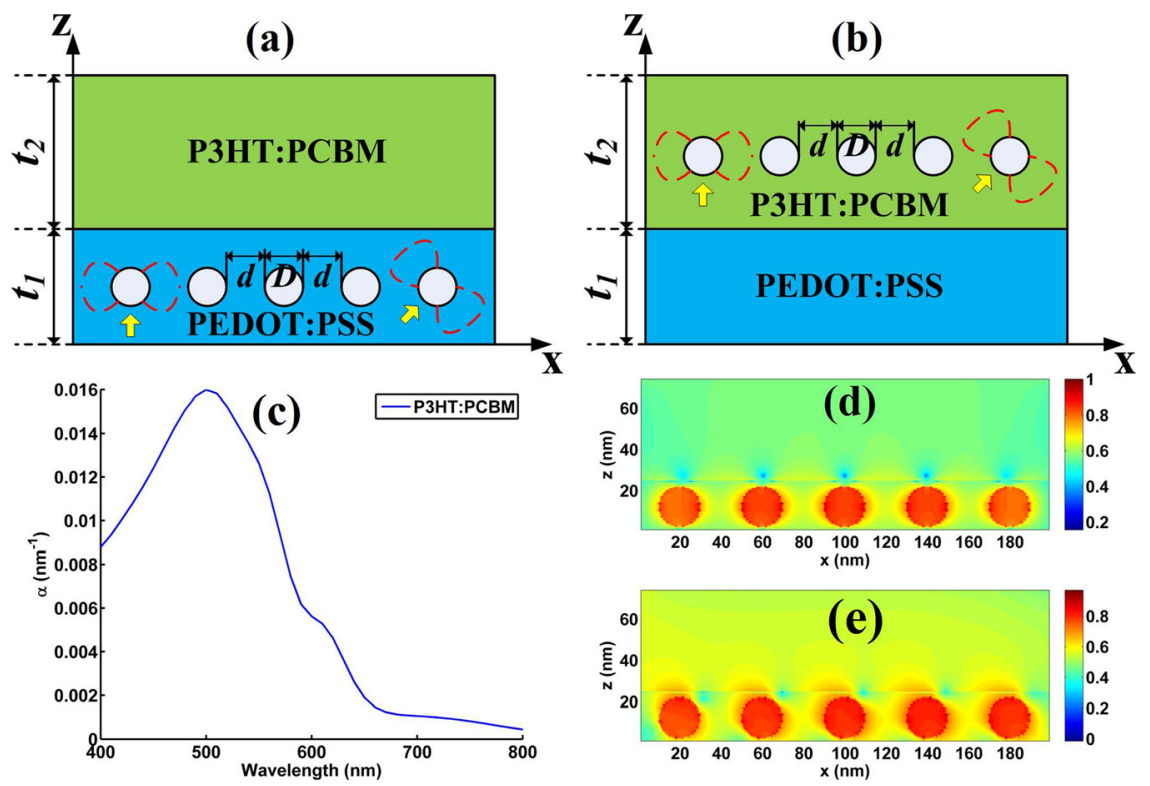

FIG. 1. (Color online) (a) and (b) A schematic OSC nanostructure. To enhance the optical absorption of the OSC, a spherical chain comprising multiple NSPs is embedded into a spacer layer (PEDOT:PSS) or into an active layer (P3HT:PCBM). The geometric size is $t_{1}=1.25 D$ and $t_{2}=2.5 D$. The spacing between adjacent NSPs is $d=D$ or $d=0.2 D$ for the separated or close-packed ones, respectively. The diameter is $D=20 \mathrm{~nm}$ or $D=40 \mathrm{~nm}$ for the small or large NSPs, respectively. The yellow arrows represent the propagation direction of an incident light with a TM polarization $\left(H_{z}^{i}=0\right)$ and the red dashed lines denote corresponding near-field profiles of NSPs. (c) Absorption coefficient of the active material. (d) and (e) Near-field polarization current distributions of the OSC nanostructure at a vertical and an oblique incidences, respectively.

$$
G=\int_{400 \mathrm{~nm}}^{800 \mathrm{~nm}} A(\lambda) \Gamma(\lambda) d \lambda
$$

where $\Gamma$ is the solar irradiance spectrum. Table I lists the total enhancement factors corresponding to Figs. 2 and 3. The total enhancement factor is the total absorption of the OSC incorporating NSPs over that excluding NSPs.

When Ag NSPs are embedded into the spacer, as the incident angle increases, both the spectral and total enhancement factors increase independent of the NSP's size and spacing. The fundamental physics is that the near-field energy of a metal nanoparticle is mainly distributed along the polarization direction of the incident $E$-field, which is critically different from the far-field scattering where the energy scatters to the propagation direction of the incident light. As shown both in Figs. 1(a) and 1(d), the concentrated
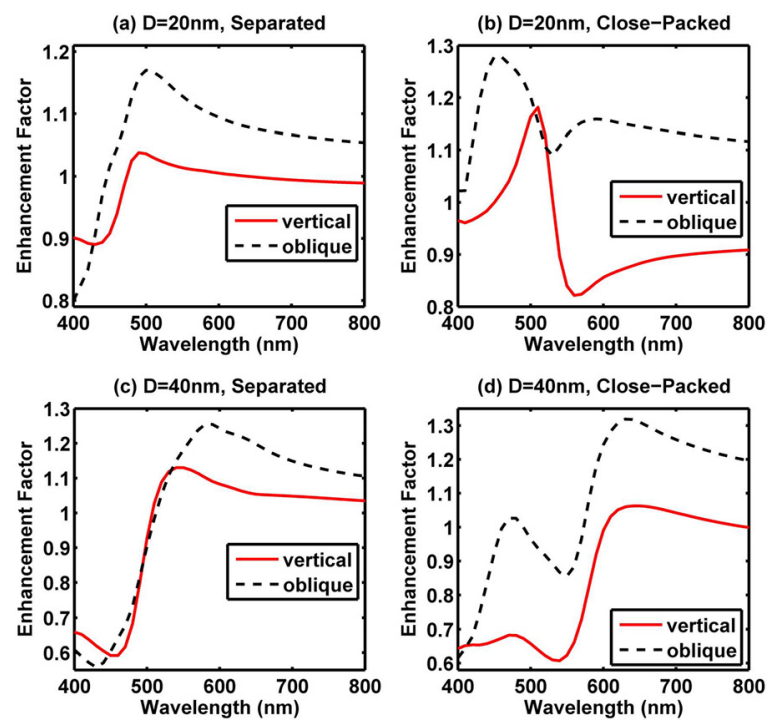

FIG. 2. (Color online) The spectral enhancement factors of the OSC with NSPs are embedded into the spacer. The angular responses of the OSC for the vertical $\left(0^{\circ}\right)$ and oblique $\left(60^{\circ}\right)$ incidences are also shown. (a) Separated small NSPs; (b) close-packed small NSPs; (c) separated large NSPs; and (d) close-packed large NSPs. electric near-field is distributed along the lateral direction at the vertical incidence, which deters the plasmonic resonance from enhancing the light absorption of the active material even though very strong near field is obtained. Hence, a careful design in introducing NSPs into a multilayer device structure is critical for enhancing the performances of plasmonic OSCs. Interestingly, absorption enhancement improves at the oblique incidence because more energy transfers to the active layer having a significant directivity as depicted in Fig. 1(e). It should be noticed that since the thickness of the active layer is thinner than one half of wavelength, near-field (not far-field) physics plays a key role in the optical design of NSPs incorporated OSCs. Compared to the spectral enhancement factors for the separated NSPs, a deep dip can be observed for the close-packed ones as shown in Figs. 2(b) and $2(\mathrm{~d})$. The dips resulting from the metallic absorption of
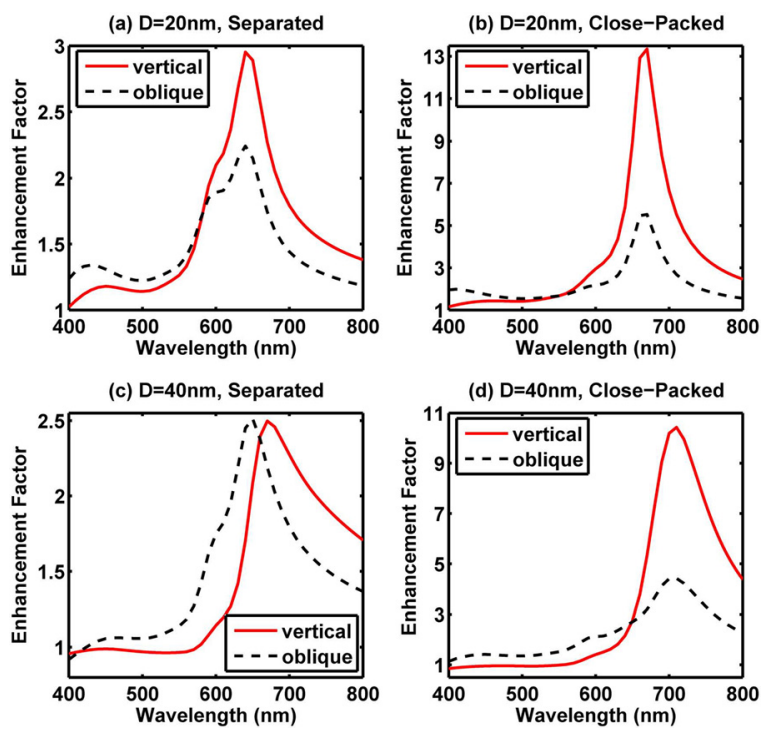

FIG. 3. (Color online) The spectral enhancement factors of the OSC with NSPs are embedded into the active layer. The angular responses of the OSC for the vertical $\left(0^{\circ}\right)$ and oblique $\left(60^{\circ}\right)$ incidences are also shown. (a) Separated small NSPs; (b) close-packed small NSPs; (c) separated large NSPs; and (d) close-packed large NSPs. 
TABLE I. Total enhancement factors for NSPs embedded into the spacer and active layer.

\begin{tabular}{lcc}
\hline \hline Spacer layer & Vertical incidence & Oblique incidence \\
\hline separated small & 0.992 & 1.078 \\
close-packed small & 0.989 & 1.174 \\
separated large & 0.927 & 0.935 \\
close-packed large & 0.725 & 0.960 \\
\hline Active layer & Vertical incidence & Oblique incidence \\
\hline separated small & 1.366 & 1.374 \\
close-packed small & 1.985 & 1.821 \\
separated large & 1.118 & 1.216 \\
close-packed large & 1.342 & 1.589 \\
\hline \hline
\end{tabular}

the NSPs correlate with the coupling resonance of the NSP chain. The close-packed NSPs, particularly for the large ones, block the light and absorb a large amount of sunlight energy. As a result, little energy penetrates into the active material. Due to the $R^{-3}$ decay of electric near-field and the reflection by the interface between the spacer and active layer, the absorption enhancement is very small when NSPs are embedded into the spacer. Comparing Fig. 2(d) to Fig. 2(b), the large close-packed NSPs with large scattering cross section have more significant enhancement away from resonance but induce deeper dips near the resonance. Weighting the two effects, the large close-packed NSPs have smaller total enhancement factor as listed in Table I.

In contrast to the NSPs embedded into the spacer, our results show that the NSPs embedded into the active layer offer stronger optical absorption, which can be observed in Table I. As seen in Fig. 1(b), the scattering energy from the NSPs is directly and sufficiently absorbed by the contiguous active material uncorrelated with the directional property of the electric near-field. Owing to the plasmon coupling and hybridization, the close-packed NSPs have more concentrated near-field distribution leading to larger enhancement (Table I). Remarkably, the absorption of the OSC has about two-fold increase by the small close-packed NSPs. For the large close-packed NSPs, the excessive red-shifted resonance reduces the spectral overlap between the resonance and the absorption peak of the active material as illustrated in Figs. 3(d) and 1(c). At the vertical incidence, the reduced spectral overlap gives a reason why the total enhancement factor by the large close-packed NSPs is smaller than that by the small ones. However, the total enhancement factor by the large close-packed NSPs increases at the oblique incidence, which distinguishes from the close-packed small NSPs. The interplay between longitudinal and transverse modes ${ }^{2}$ supported by the NSP chain is a physical origin of the phenomenon. Having larger geometric size and stronger retardation effect, the large close-packed NSPs support more red-shifted longitudinal modes at the vertical incidence and more blue-shifted transverse modes at the oblique incidence (See Figs. 3(b) and 3(d)). In comparison with the red-shifted longitudinal modes, the blue-shifted transverse modes have a better spectral overlap with the absorption coefficient of the active material and can be further exploited or engineered in a future design of OSCs.
Regarding the electrostatic limit described by the Laplace equation, the near-field or far-field response of a subwavelength scatterer is independent of the scatterer's size and depends only on its shape. Therefore, it may cause a misunderstanding that the same enhancement can be obtained if the scaling ratio of a device structure to a concentrator remains constant. However, using the same scaling ratio as shown in Figs. 1(a) and 1(b), we find that the large NSPs and small ones have noticeable differences both in the spectral and total enhancement factors. The breakdown of the scaling law can be explained by the retarded and multiscale effects. The electromagnetic response of a single NSP is dominated by the electrostatic (nanocircuit) physics, but that of multiple NSPs are governed by the electrodynamic (wave) physics with nonnegligible retardation and long-range interplay between each NSP. Furthermore, large-scale OSC nanostructure and smallscale NSPs strongly couple with each other, which makes the optical path very complicated, and the trapping confinement, together with leaky loss, must be considered quantitatively.

In conclusion, we study the near-field multiple scattering effects of plasmonic NSPs embedded into the thin-film OSC. The absorption enhancement of the OSC strongly depends on the directional property of near-field scattering from NSPs and the interplay between longitudinal and transverse modes supported for the NSPs embedded into the spacer and active layer, respectively. Moreover, the complex coupling between NSPs and device makes the scaling law in electrostatics inapplicable. The work provides the fundamental physical understanding and design guidelines for a typical class of plasmonic photovoltaics.

The authors acknowledge the support of the grants (Nos. 712010, 711609, and 711511) from the Research Grant Council of the Hong Kong. This project is also supported in part by a Hong Kong UGC Special Equipment Grant (SEG HKU09).

${ }^{1}$ K. L. Chopra, P. D. Paulson, and V. Dutta, Prog. Photovoltaics 12, 69 (2004).

${ }^{2}$ S. A. Maier, Plasmonics: Fundamentals and Applications (Springer, New York, 2007).

${ }^{3}$ R. B. Konda, R. Mundle, H. Mustafa, O. Bamiduro, A. K. Pradhana, U. N. Roy, Y. Cui, and A. Burger, Appl. Phys. Lett. 91, 191111 (2007).

${ }^{4}$ S. H. Lim, W. Mar, P. Matheu, D. Derkacs, and E. T. Yu, J. Appl. Phys. 101, 104309 (2007).

${ }^{5}$ P. Matheu, S. H. Lim, D. Derkacs, C. McPheeters, and E. T. Yu, Appl. Phys. Lett. 93, 113108 (2008).

${ }^{6}$ A. J. Morfa, K. L. Rowlen, T. H. Reilly, M. J. Romero, and J. van de Lagemaat, Appl. Phys. Lett. 92, 013504 (2008).

${ }^{7}$ K. R. Catchpole and A. Polman, Appl. Phys. Lett. 93, 191113 (2008).

${ }^{8}$ M. J. Mendes, A. Luque, I. Tobias, and A. Marti, Appl. Phys. Lett. 95, 071105 (2009).

${ }^{9}$ Y. A. Akimov, W. S. Koh, and K. Ostrikov, Opt. Express 17, 10195 (2009).

${ }^{10}$ J.-Y. Lee and P. Peumans, Opt. Express 18, 10078 (2010).

${ }^{11}$ D. D. S. Fung, L. F. Qiao, W. C. H. Choy, C. C. D. Wang, W. E. I. Sha, F. X. Xie, and S. L. He, "Optical and Electrical Properties of Efficiency Enhanced Polymer Solar Cells with Au Nanoparticles in PEDOT-PSS Layer", J. Mater. Chem. (in press).

${ }^{12}$ H. A. Atwater and A. Polman, Nat. Mater. 9, 205 (2010).

${ }^{13}$ A. D. Rakic, A. B. Djurisic, J. M. Elazar, and M. L. Majewski, Appl. Opt. 37, 5271 (1998).

${ }^{14}$ See supplementary material at http://dx.doi.org/10.1063/1.3638466 for numerical implementation of volume integral equation method. 